\title{
Background in the search for dark photon in $e^{+} e^{-}$annihilation
}

\author{
Venelin Kozhuharov ${ }^{1,2, a}$ \\ ${ }^{1}$ University of Sofia "St. KI. Ohridski", 5 J. Bourchier Blvd., 1164 Sofia, Bulgaria \\ ${ }^{2}$ Laboratori Nazionali di Frascati, 00044 Frascati RM, Italy
}

\begin{abstract}
An important ingredient in the search for new particles and rare phenomena is the correct estimation of the expected contribution from processes originating from the Standard Model. The search for a new vector degree of freedom, the Dark photon, is affected by the precise knowledge of the electromagnetic interactions. In the case of invisibly decaying Dark photon produced in $e^{+} e^{-}$annihilation the evaluation of the background should be performed with less than $1 \%$ uncertainty since the sensitivity of the proposed experiments depend lineary on it. The present status of the background estimation and the considered processes are reviewed and discussed, with a special attention devoted to the possible unresolved issues.
\end{abstract}

\section{Introduction}

The nature of Dark Matter (DM) and its possible interaction with the Standard Model (SM) particles is still one of the unresolved puzzles in nowadays physics. A framework in which a new plethora of particles almost completely decoupled from the known degrees of freedom could exist gained attention with the possibility to address few of the existing "smoking guns" in particle physics simultaneously - the muon anomalous magnetic moment [1], the excess of antimatter in cosmic rays [2-5], the annual modulation observed in the DAMA/Libra data [6], and the ${ }^{8} \mathrm{Be}$ anomaly $[7,8]$. This hidden sector may be connected to the SM particles through a portal - a single messenger that exhibits non-vanishing interactions with the particles both in the visible and in the hidden sector. The most exploited scenario is the case of a new vector field, a so called Dark photon (DP) $A^{\prime}$, which could be the mediator of an additional gauge symmetry, U(1) in the simplest case. Some of the SM particles could be charged under the new interaction or the coupling between them and $A^{\prime}$ could be generated dynamically through the kinetic mixing of $A^{\prime}$ with any of the neutral electroweak gauge fields. In the most simple case the interaction strength $\alpha^{\prime}$ could be normalized to the electromagnetic interaction strength $\alpha_{E M}$, thus leaving only two new parameters $-\epsilon^{2}=\alpha^{\prime} / \alpha_{E M}$ and the mass of the dark photon $m_{A^{\prime}}$.

The dark sector may contain extra degrees of freedom, $\chi \mathrm{s}$, with mass $m_{\chi}$ which couple to $A^{\prime}$ without suppression, with a relative strength $\alpha_{D}$. Depending on the mass hierarchy in the invisible sector, the phenomenology of the Dark photon events could be divided into two well distinctive scenarios:

- Visibly decaying $A^{\prime}$ : If $m_{\chi}>m_{A^{\prime}}$ then the dark photon decays to SM particles with a rate proportional to $\epsilon^{2}$. For

\footnotetext{
a e-mail: Venelin.Kozhuharov@ cern.ch
}

$m_{A^{\prime}}<2 m_{\mu}$ the only allowed final state is the $e^{+} e^{-}$. The experiments addressing this scenario contain high resolution spectrometers to measure the electron/positron momenta and to reconstruct the $A^{\prime}$ decay vertex.

- Invisible $A^{\prime}$ : If $m_{A^{\prime}}>2 m_{\chi}$ then the dominant decay process will be $A^{\prime} \rightarrow \chi \chi$ since it is not suppressed by the small factor $\epsilon$. Since in most of the cases $\chi$ s can not be detected the $A^{\prime}$ experimental signature will be events with missing mass or missing energy in the final state.

No DP has been observed so far and the present data put constrain in the DP parameter space in the range $\epsilon^{2}<10^{-5}-10^{-6}$ for $m_{A^{\prime}}>5 \mathrm{MeV}$. However, most of the experiments have addressed only the visibly decaying $A^{\prime}$, thus leaving the parameter space for invisible $A^{\prime}$ scenario largely unexplored.

A huge variety of Dark photon models exist [9], including leptophylic, leptophobic, protophobic [10, 11] and many others may appear. The extremely rich possible phenomenology points towards the necessity of execution of a diverse experimental program with novel techniques, allowing to probe different regions in the parameter space that might even seem currently excluded in some of the simplest cases by a particular process.

\section{Invisible dark photon searches}

When the DP is quasi-stable (for small $\epsilon^{2}$ ) or decays into $\chi \chi$ the full reconstruction of the event by measuring only the final state particles is impossible. To overcome this problem precise knowledge of the initial state is necessary. One of the most suitable processes to search for an associate $A^{\prime}$ production in annihilation, $e^{+} e^{-} \rightarrow A^{\prime} \gamma$, where both the electron and the positron are with known parameters. This can be realized with the positron-on-target technique where the positrons from an accelerator impinge on 


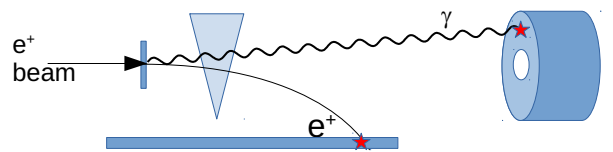

Bremsstrahlung

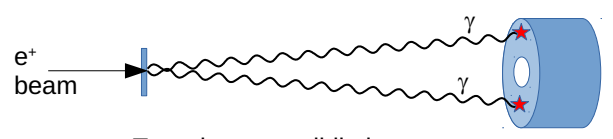

Two photon annihilation

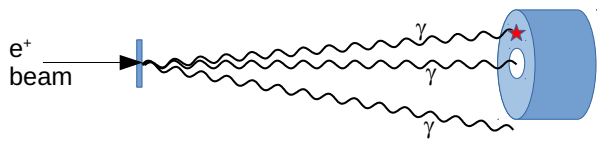

Three photon annihilation

Figure 1. Dominant processes contributing to the search for invisible dark photon production in $e^{+} e^{-}$annihilation

a thin target and have the possibility to annihilate in flight with the matter electrons. A key ingredient for such an experiment is an electromagnetic calorimeter which should measure the properties of the recoil photon.

The single kinematics quantity, the missing mass, is reconstructed according to

$$
M_{m i s s}^{2}=\left(P_{e+}+P_{e-}+P_{\gamma}\right)^{2}
$$

where $P_{e+}$ is taken from the known beam momentum, $P_{e-}$ is assumed as $\left(m_{e}, 0,0,0\right)$ (electrons are taken to be at rest, compared to the much higher beam energy) and $P_{\gamma}$ is calculated using the measured energy $E_{\gamma}$ and the impact point of the accompanying photon in the electromagnetic calorimeter.

Currently three experiments, PADME [12, 13], VEPP3 $[14,15]$, and MMAPS [16] are planning to exploit this technique to search for possible Dark Photon production in the annihilation process. Their parameters are listed in table 1. Two of them, PADME and VEPP3, got approved and are in construction phase.

The dominant background contributions together with the possible approach to suppress them are shown in figure 1 and are:

- Radiative emission of photons. The average angle of photon emission with respect to the positron beam direction falls as $1 / E_{\text {beam }}$. Most of the high energy photons are focused in the very forward direction. Since the occupancy of the central part of the electromagnetic calorimeter may reach intolerable values, usually it is made as a ring-shaped structure without any active material close to the beam axis. The only way to suppress the bremsstrahlung background is the addition of a detector able to measure the emitting positron and perform matching with the detected in the calorimeter photon. This could be done by implementing a magnetic field

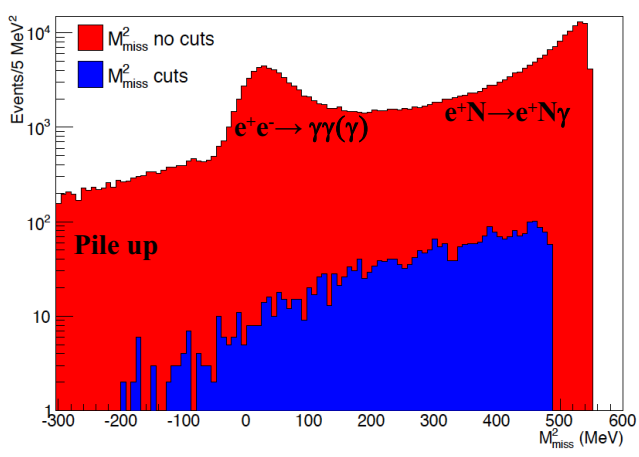

Figure 2. Missing mass squared distribution of the dominant background events, obtained with the simulation of the PADME experiment, based on GEANT4

and a charged particle detector, which may also provide momentum measurement.

- Two photon annihilation. The missing mass distribution for $e^{+} e^{-} \rightarrow \gamma \gamma$ events, when only a single photon is detected, peaks at zero. However, due to symmetric realization of the calorimeter and its central hole, the acceptance for single photon events from two photon annihilation is almost negligible.

- Multiphoton annihilation. The effect of the presence of additional photons in the annihilation process has to be considered when the energy of the extra photons is higher than the beam energy spread or the energy resolution of the calorimeter. These extra photons break the symmetry of the event and decrease the vetoing capability of the cluster multiplicity in the calorimeter. In addition, the missing mass distribution for such events does not peak at zero which further decreases their suppression.

The cross sections for each of these processes are listed in table 2

A typical missing mass distribution spectrum, obtained with a full GEANT4 [17] simulation of the PADME setup, is shown in figure 2.

The signal region is defined as

$$
\left(m_{A^{\prime}}^{2}-x \sigma_{m_{A^{\prime}}^{2}}\right)<M_{m i s s}^{2}<\left(m_{A^{\prime}}^{2}+x \sigma_{m_{A^{\prime}}^{2}}\right),
$$

where $\sigma_{m_{A^{\prime}}^{2}}$ depends on $m_{A^{\prime}}$ and is obtained from Monte Carlo simulation of the expected signal while $x$ usually takes values between 1 and 2 . As can be seen from figure 2 , the search for DP in annihilation process is not a zero background search. For most of the $m_{A^{\prime}}$ intervals the expected background contribution from SM processes in the defined signal region is of the order $O\left(10^{4}-10^{5}\right)$ events.

The minimal accessible relative interaction strength of the DP $\epsilon_{\min }^{2}$ of the experiment is proportional to the the uncertainty of the estimation of the observed potential signal, and is inversely proportional to the product of the detection 
Table 1. Proposed experiments to search for the production of Dark photons in $e^{+} e^{-}$annihilation.

\begin{tabular}{lccc}
\hline & PADME & MMAPS & VEPP3 \\
\hline Place & LNF & Cornell & Novosibirsk \\
Beam energy & $550 \mathrm{MeV}$ & $6 \mathrm{GeV}$ & $500 \mathrm{MeV}$ \\
$m_{A^{\prime}}$ limit $[\mathrm{MeV}]$ & 23.7 & 74 & 22.6 \\
Target thickness & $2 \times 10^{22} e^{-} / \mathrm{cm}^{2}$ & $\mathrm{O}\left(10^{23}\right) e^{-} / \mathrm{cm}^{2}$ & $5 \times 10^{15} e^{-} / \mathrm{cm}^{2}$ \\
Beam intensity $[\mathrm{mA}]$ & $8 \times 10^{-11}$ & $2.3 \times 10^{-6}$ & 30 \\
$e^{+} e^{-} \rightarrow \gamma \gamma$ rate & 15 & $2.2 \times 10^{6}$ & $1.5 \times 10^{6}$ \\
$\epsilon^{2}$ limit (plateau) & $10^{-6}\left(10^{-7} \mathrm{SES}\right)$ & $10^{-6}-10^{-7}$ & $10^{-7}$ \\
Time scale & $2017-2018$ & $?$ & 2020 \\
Status & Approved & Funds identification & Approved \\
\hline
\end{tabular}

Table 2. Dominant background contributions to the search for the production of Dark photons in $e^{+} e^{-}$annihilation. Cross sections are calculated for $E_{\text {beam }}=550 \mathrm{MeV}$ and carbon target.

\begin{tabular}{|l|c|c|}
\hline Process & $\sigma[\mathrm{mb}]$ & Comment \\
\hline \hline$e^{+} e^{-} \rightarrow \gamma \gamma$ & 1.55 & \\
$e^{+} N \rightarrow e^{+} N \gamma$ & 4000 & $E_{\gamma}>1 \mathrm{MeV}$ \\
$e^{+} e^{-} \rightarrow \gamma \gamma \gamma$ & 0.16 & $E_{\gamma}>1 \mathrm{MeV}$ \\
$e^{+} e^{-} \rightarrow e^{+} e^{-} \gamma$ & 188 & $E_{\gamma}>1 \mathrm{MeV}$ \\
\hline
\end{tabular}

efficiency and the two photon annihilation statistics, which is used to determine the total particle flux.

$$
\epsilon_{\min }^{2} \sim \sigma_{t o t} /\left(A c c\left(A^{\prime}\right) \times N(\gamma \gamma)\right)
$$

$\operatorname{Acc}\left(A^{\prime}\right)$ depends on the design of the experiment and $(\gamma \gamma)$ is determined from the target and beam time.

Since the number of the signal events is just the difference between the expected and the observed number of events, $N_{\text {signal }}=N_{o b s}-N_{\text {exp }}$, the total uncertainty of the potential signal is given by

$$
\sigma_{\text {tot }} \sim\left(\sqrt{N_{\text {obs }}}\right) \oplus\left(\delta_{\text {model }} \times N_{\text {exp }}\right),
$$

assuming that the number of the expected events can be determined with high accuracy through Monte Carlo generation of large samples so that the statistics factor in $\sigma\left(N_{\text {exp }}\right)$ vanishes. The model uncertainty, $\delta_{\text {model }}$, represents the accuracy of the simulation of a given background process. For large statistics experiments the term $\delta_{\text {model }} \times N_{\text {exp }}$ becomes dominant in the total uncertainty determination.

In the particular case of PADME, the relative statistical uncertainty in the number of observed events is expected to be below $1 \%$. A short review of the current status of the precision of the simulation of the different background sources follows.

\section{Bremsstrahlung background}

In the positron-on-target Dark photon search the positrons do not impinge the target equally spaced in time but are rather bunched in groups with a length of picoseconds to nanoseconds. Only in the long bunch limit or infinite time resolution the experiments turn into single particle measurement. An event is defined by the time resolution of the detectors and usually incorporates $\mathrm{O}\left(10^{3}-10^{4}\right)$ positrons interacting in the target. Since the total cross section for bremsstrahlung emission is high (see table 2) each event contains positrons which have undergone significant energy loss in the target. These positrons could be detected and vetoed to suppress the background from bremsstrahlung events, when a single cluster in the calorimeter with $E_{\gamma}$ is identified. However, when applying this technique, in order to keep the acceptance for $A^{\prime}$ detection at the level of $\mathrm{O}(20 \%)$, it is necessary to match the sum of the positron energy $E_{e+}$ and the photon energy $E_{\gamma}$ and veto only events for which

$$
\left|E_{e+}+E_{\gamma}-E_{\text {beam }}\right|<E_{\text {cut }},
$$

where $E_{\text {cut }}$ is determined by the resolution.

The total cross section for the emission of a photon with energy $k$ by an electron or positron when passing through matter can be written as [17]

$$
\frac{d \sigma}{d k}=\frac{d \sigma_{n}}{d k}+Z \frac{d \sigma_{e}}{d k}
$$

where $d \sigma_{n} / d k$ is the differential cross section for bremsstrahlung emission in the field of the nucleus and $Z d \sigma_{e} / d k$ accounts for bremsstrahlung produced in the field of $Z$ atomic electrons.

A common approach for the estimation of the background contribution due to bremsstrahlung is the usage of GEANT4 simulation package. Below $1 \mathrm{GeV}$ electron energy, the cross section for bremsstrahlung from neutral atoms in the GEANT4 versions 9.5 and above [18] is obtained from the interpolation of the Seltzer and Berger tabulated data of $d \sigma_{n} / d k$ and $d \sigma_{e} / d k$ [19]. This model shows good agreement with the experimental data. However, the uncertainty for $d \sigma / d k$ for electrons with energy $50 \mathrm{MeV}$ $<E_{e^{+}}<1 \mathrm{GeV}$ is in the range (3-5)\%. This uncertainty is few times higher than the expected statistical uncertainty in the determination of the observed number of events $N_{o b s}$ and additional tuning of the model parameters might be necessary to reproduce the observed bremsstrahlung spectrum. For positrons with energy higher than $1 \mathrm{GeV}$, an analytic expression for $d \sigma / d k$, including also the LandauPomeranchuk-Migdal effect, is used in GEANT4.

The experiments searching for Dark photon in annihilation employ targets with thickness of the order and below $100 \mu \mathrm{m}$. Usually at such distance the energy loss of the traversing particles is sampled in a single step leading to the physical generation of at most a single emit- 


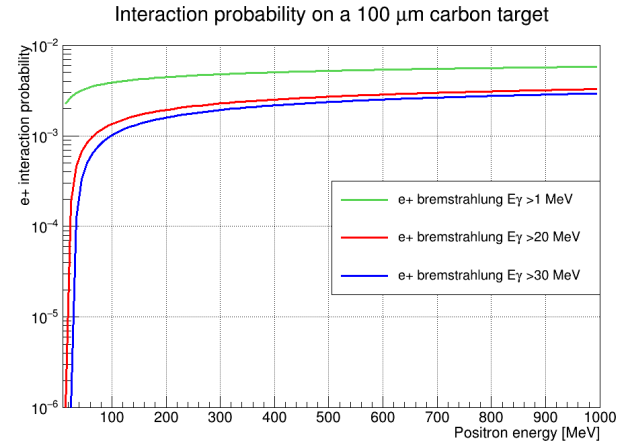

Figure 3. Probability for emission of a bremsstrahlung photon with energy $E>E_{\text {cut }}$ as a function of the positron energy. The cross sections are calculated using the GEANT4, version 9.4 parametrized model for bremsstrahlung emission.

ted photon. Although the targets are thin, the probability for the emission of a photon with energy $E_{\gamma}>20 \mathrm{MeV}$ for $100 \mu \mathrm{m}$ carbon target traversed by a positron with energy $550 \mathrm{MeV}$ is $0.28 \%$, as can be seen from figure 3. Since the dependence on the beam energy for 300 $\mathrm{MeV}<E_{\text {beam }}<600 \mathrm{MeV}$ is weak, naively, the probability for the emission of two photons with $E_{\gamma}>20 \mathrm{MeV}$ would be $\sim 10^{-5}$. Although quite low, this probability is not negligible since the $e^{+} N \rightarrow e^{+} N \gamma \gamma$ events may not satisfy the relation in equation (5), when a single photon is detected. Such second order effects might be treated in a dedicated way, by forcing their generation explicitly and merging them with the full sample used for background estimation, applying an appropriate normalization factor. In any case, the proper background estimation due to multiple bremsstrahlung emission in the target is important.

In the positron-on-target experiments the bremsstrahlung background can be estimated with electron beam with the same geometrics and kinematics characteristics as the nominal positron beam. Ideally, the acquired statistics should be an order of magnitude higher than in the nominal running mode with positrons. In this way only the uncertainty due to the additional factor, which accounts for the difference in the interactions of positron and electrons, remains. The electron data can also be exploited to measure the bremsstrahlung cross section $d \sigma / d k$ for the target materials and to tune the GEANT4 parameters.

\section{Annihilation background}

The cross section for in flight annihilation of the positron is described by the Heitler formula [20]

$$
\begin{array}{r}
\sigma(E, Z)=\frac{Z \pi r_{e}^{2}}{\gamma+1} \times \\
{\left[\frac{\gamma^{2}+4 \gamma+1}{\gamma^{2}-1} \ln \left(\gamma+\sqrt{\gamma^{2}-1}\right)-\frac{\gamma+3}{\sqrt{\gamma^{2}-1}}\right],}
\end{array}
$$

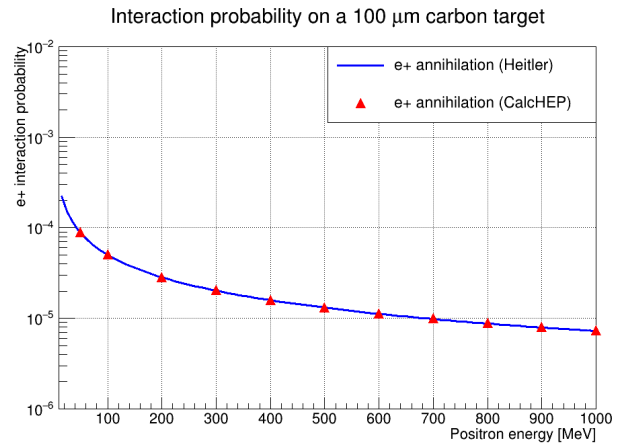

Figure 4. Comparison of the two photon annihilation probability for a positron traversing $100 \mu \mathrm{m}$ thich carbon target calculated with CalcHEP and according to the Heitler formula.

where $E$ is the positron energy, $r_{e}$ is the classical electron radius and $\gamma$ is the Lorentz factor. The electron is considered to be at rest. This process is included in the default GEANT4 electromagnetic library and is taken into account when propagating positrons through matter. However, this is not the case with the $e^{+} e^{-}$annihilation into three or more photons, which are completely ignored. While the symmetric design of the calorimeters of PADME, VEPP3, and MMAPS efficiently suppresses the two photon annihilation, the production of extra photons in the final state might result in the detection only of a single particle in the calorimeter. Then the invariant mass of the undetected particles will be reconstructed as missing mass for the event, $M_{m i s s}^{2}=\left(\sum P_{\text {miss }}^{\gamma}\right)^{2}$. Such multiphoton events with just a single detected photon represent an irreducible background to the search for Dark photon, leaving the only possibility to estimate their contribution precisely.

The cross section for three photon annihilation was calculated with CalcHEP - a tool allowing to calculate Feynman diagrams and to perform integration over a multiparticle phase space [21]. The CalcHEP model was updated to account for the non-zero electron mass and the values of the interaction constants at low energy were introduced. The difference between the the calculated with CalcHEP cross section for $e^{+} e^{-} \rightarrow \gamma \gamma$ and the analytic expression in equation (7) is shown in figure 4. It was less than $1 \%$ for positron energy in the interval $100 \mathrm{MeV}$ $<E_{e^{+}}<1 \mathrm{GeV}$ and proved the applicability of CalcHEP for this low energy regime.

The divergency in $\sigma\left(e^{+} e^{-} \rightarrow 3 \gamma\right)$ when $E_{\gamma} \rightarrow 0$ is cancelled by the virtual photon corrections to the $\sigma\left(e^{+} e^{-} \rightarrow\right.$ $2 \gamma$ ). However, experimentally it is easier to introduce a cut on $E_{\gamma}$ below which any sensitivity to extra photons is lost - this cut is determined by the beam energy spread. For $E_{e^{+}}=550 \mathrm{MeV}$ and $E_{\gamma}>5 \mathrm{MeV}$, the relative contribution of the $3 \gamma$ annihilation to the $2 \gamma$ cross section was estimated to be of the order of $4.5 \%$, as can be obtained from figure 5 . This value increases gradually with the positron beam energy. In the case of a single ring shaped calorimeter, the three photon annihilation turns to be the dominant 


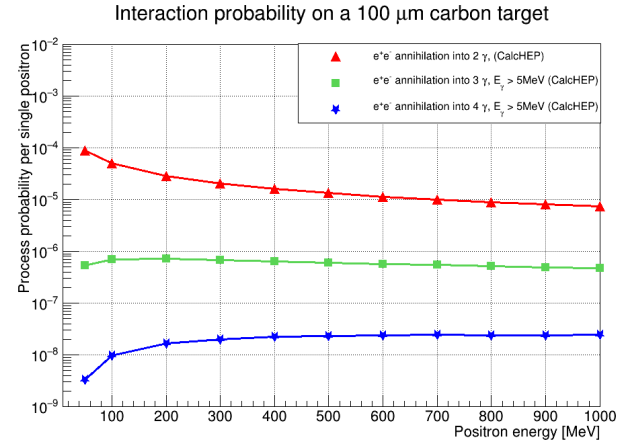

Figure 5. Probability for multiphoton production through annihilation of positrons on $100 \mu \mathrm{m}$ carbon target into photons with energy $E>5 \mathrm{MeV}$, as a function of the positron energy. The cross sections are calculated with CalcHEP.

background contribution to the dark photon search in the intermediate $m_{A^{\prime}}$ region.

Proceeding further, the total annihilation width $\Gamma\left(e^{+} e^{-} \rightarrow N \gamma\right)$ is given by the sum of the different possible final states

$$
\begin{aligned}
\Gamma_{\text {annih }}= & \Gamma\left(e^{+} e^{-} \rightarrow \gamma \gamma\right)+\Gamma\left(e^{+} e^{-} \rightarrow \gamma \gamma \gamma\right)+ \\
& \Gamma\left(e^{+} e^{-} \rightarrow 4 \gamma\right)+\ldots \approx 1.05 \times \Gamma\left(e^{+} e^{-} \rightarrow \gamma \gamma\right) .
\end{aligned}
$$

For $E_{e^{+}} \approx 500 \mathrm{MeV}$ the value differs from the Born approximation by as much as $5 \%$.

To estimate the background from three photon annihilation events a dedicated sample generation might be attempted. As in the multi bremsstrahlung case, this sample has to be merged with the full background sample, after applying a factor to account for the three photon annihilation cross section. However, since the four photon contribution to the three photon sample is of the same order as of the three photon to the two photon, currently the uncertainty of this scale factor is of the order of $5 \%$, as seen from figure 5. This uncertainty is again few times higher than the expected $\sigma\left(N_{o b s}\right)$.

A different approach is to use dedicated Monte Carlo event generators, which take into account the radiative corrections in the proper way. Two of them are currently available, BABAYGA [22, 23] and MCGPJ [24], but at least one of them has to be modified to include the mass of the electron due to the small invariant mass regime, $\mathrm{O}(10$ $100 \mathrm{MeV}$ ), of the positron-on-target experiments searching for Dark photons.

\section{Conclusions}

The correct and precise estimation of the background contribution is crucial for the interpretation of the results of the experiments aiming at a search for Dark photon production in positron-on-target annihilation. The required precision is of the order of $1 \%$ and below. Since the uncertainty in the models used to simulate the background may extend up to $5 \%$ a more detailed investigation is necessary.
A dedicated measurement of the differential cross section for radiative emission of photons by positrons and electrons with low energy $(\mathrm{O}(10-100 \mathrm{MeV}))$ in the field of the nuclei and the atomic electrons is highly desirable and will allow to perform the tuning of the existing Monte Carlo simulation tools. A possible alternative for the positron-on-target annihilation experiments is to exploit also data driven methods for background estimation, which may include acquiring data with electron beam, keeping the conditions unchanged. In addition, the multiphoton annihilation cross section, even if pure electromagnetic process, has to be known to the percent level and may also require experimental measurements for the tuning of the Monte Carlo generators.

\section{Acknowledgements}

This work has been partially supported by LNF-INFN under grant LNF-SU 70-06-497/07-10-2014 and NSFBulgaria under grant MON-FNI DN08-14/14.12.2016.

\section{References}

[1] G. W. Benett et al. (The g-2 Collaboration), Phys. Rev. D73, 072003 (2006).

[2] O. Adriani et al. [PAMELA Collaboration], Nature 458, 607 (2009).

[3] M. Ackermann et al. [Fermi LAT Collaboration], Phys. Rev. Lett. 108, 011103 (2012).

[4] M. Aguilar et al. [AMS Collaboration], Phys. Rev. Lett. 110, 141102 (2013).

[5] M. Aguilar et al. [AMS Collaboration], Phys. Rev. Lett. 117, no. 9, 091103 (2016).

[6] R. Bernabei et al., Eur. Phys. J. C67, 39 (2010).

[7] A.J. Krasznahorkay et al., Phys. Rev. Lett. 116, 042501 (2016).

[8] A.J. Krasznahorkay, talk at ADMPP, Messina, 24-27 October, Italy (2016).

[9] M. Raggi and V. Kozhuharov, Riv. Nuovo Cim. 38, no. 10, 449 (2015).

[10] J. L. Feng, B. Fornal, I. Galon, S. Gardner, J. Smolinsky, T. M. P. Tait and P. Tanedo, Phys. Rev. Lett. 117, no. 7, 071803 (2016)

[11] I. Galon, talk at ADMPP, Messina, 24-27 October, Italy (2016).

[12] M. Raggi and V. Kozhuharov, Adv. High Energy Phys. 2014, 959802 (2014)

[13] M. Raggi, talk at ADMPP, Messina, 24-27 October, Italy (2016).

[14] B. Wojtsekhowski, D. Nikolenko and I. Rachek, arXiv:1207.5089 [hep-ex].

[15] I. Rachek et. al, talk at ADMPP, Messina, 24-27 October, Italy (2016).

[16] J. Alexander, talk at ADMPP, Messina, 24-27 October, Italy (2016).

[17] S. Agostinelli et al. [GEANT4 Collaboration], Nucl. Instrum. Meth. A 506, 250 (2003). 
[18] V. Ivantchenko, talk at ADMPP, Messina, 24-27 October, Italy (2016).

[19] S.M.Seltzer and M.J.Berger. Nucl.Inst.Meth. B12, 95 (1985).

[20] W. Heitler., The Quantum Theory of Radiation, Clarendon Press, Oxford (1954).

[21] A.Pukhov et al., Preprint INP MSU 9841/542,arXiv:hep-ph/9908288
A.Pukhov, e-Print Archive: hep-ph/0412191.

[22] C. M. Carloni Calame, Phys. Lett. B 520, 16 (2001).

[23] C. M. Carloni Calame, talk at ADMPP, Messina, 2427 October, Italy (2016).

[24] A. B. Arbuzov, G. V. Fedotovich, F. V. Ignatov, E. A. Kuraev and A. L. Sibidanov, Eur. Phys. J. C 46, 689 (2006). 